\title{
数字化测绘技术在工程测量中的应用分析
}

徐苹

贵州省黔西南州安龙县国土资源局

DOI:10.32629/gmsm.v1i3.41

[摘 要] 现如今,计算机技术以及网络信息技术实现了快速的发展,在工程测量这一问题上,数字化测绘技术也得到了越来越 广泛的应用。现如今的工程测量工作如果单纯的依靠传统的测绘技术已经不能够满足整个工程对数据精准度的要求,所以有 效地依靠数字化测绘技术对工程测量中高精准度的数据获取也具有非常重要的作用。任何一项工程与科技化,信息化进行结 合, 都能够大幅度的提升工作的效率, 所以数字化测绘技术应运而生。我国近年来在工程测量中对数字化测绘技术实现了越来 越广泛的应用, 但是依然存在着非常大的进步空间。本篇文章便对数字化测绘技术在工程测量中的应用进行了简要的探讨, 意 在研究数字化测绘技术在工程测量中实现更广泛的应用, 从而提升工作的效率。

[关键词] 数字化测绘技术; 工程测量; 应用分析

\section{1 数字化测绘技术的特征分析}

1.1 对 GIS 系统提供的信息源进行合理充分的运用

GIS 系统能够为后期的图纸绘制和设计提供准确, 真实 的数据, 在现如今工程测量这一环节上, 我国大部分工程单 位均未实现与 GIS 系统的直接衔接, 所以 GIS 系统在数字化 测绘技术的发展过程中依然存在着非常大的进步空间, 但是 现如今科技发展的速度有目共睹, 在未来, GIS 系统与数字化 测绘技术实现无缝衔接也是很有可能的。在工程测量的过程 中很有可能会遇到空间跨度较大的领域, 所以数据的收集也 具有一定的难度, 那么在这种情况下, GIS 系统所发挥的作用 便是十分重要的, GIS 系统可以有效的收集比例过大的空间 数据, 从而为后期的实地工程测量提供准确的数据源。

1.2 实现测图的自动化

数字化测绘技术能够对工程测量这一过程实现有效的 自动化控制, 从而大幅度的减少人力资本的应用, 减少建筑 工程施工单位的运行成本, 对施工单位的经济效益提升也具 有一定的辅助作用。在整体的图画绘制这一过程中可以利用 非常专业的软件对土地工程测量实现自动化的计算, 对识别 和分辨从而进行选择等也具有一定的辅助作用。相比于传统 条件下的手动绘图来说, 自动化绘图能够大幅度的提升绘图 的效率, 并且在保证高效率的同时也能够保证准确度较高的 特性, 对工程测量以及绘图环节等均有十分重要的辅助作用, 同时也有利于绘图的更加规范和准确, 避免人为的错误, 从 而有效的保证绘图的准确性。

\section{3 有效提高测图的精准度}

工程测量这一环节对于数据源的准确性具有十分明显 和严格的要求, 有效地利用数字化测绘技术, 将数字智能技 术与工程测量进行有效的结合, 能够大幅度的提升数据源的 精确度。前文我们已经提到, 现如今人们对工程的质量等提 出了越来越高的要求, 所以工程测量的测图准确度也具有十 分重要的现实意义。单单的依靠传统的测绘技术来进行工程 的测量已经不能够满足现如今工程测量所提出的越来越高
的要求, 而数字化的测绘技术能够有效地减少地图测绘的数 据误差, 从而为后期工程测量提供一组准确的数据, 避免后 期由于数据不准确造成的资金成本等大幅度的损失。

\section{4 完整地反映信息, 图形属性也较为丰富}

数字化测绘技术不仅能够有效地保证数据源的精确性, 同时还能够有效地保证坐标的完整性, 同时对图形所包含的 信息也能够以较丰富的手段进行有效的展示, 在图形绘制完 毕之后, 如果将测绘点上的编码信息进行有效的连接, 也可 以获取到整个计算机数据库中的相对应的符号, 从而对地形 等进行有效的判断和分析, 在后期工程测量中也能够发挥相 关的作用, 对信息的检索等环节也具有十分重要的意义。

\section{2 数字化测绘技术在工程测量中的应用}

2.1 在数字地球中的应用

测绘方案种类众多, 其中数字地球测绘是现如今工程测 量以及数字化测绘技术在绘图应用中一种较常用的测绘方 案, 同时也可以说, 数字地球测绘是一种非常常用的测绘技 术, 此种测绘技术与传统的测绘技术相比, 最大的优势在于 所获得的数据信息与以往相比更全面, 更具有综合性, 除了 能够获得传统测绘技术所获取到的地理信息和其他相关的 气候, 水土等信息之外, 还能够在此基础上建立科学合理的 二维平面和三维空间结构, 所以在此结构的应用之中, 才能 够对当地的环境进行综合充分的了解, 并且从社会经济和环 境等多方面能够充分的了解当地的建筑环境, 从而对后期地 区数字地球测绘方案提供有效的数据支持, 不仅能大幅度的 提升后期土地工程测量的效率, 还能够有效地保证测量工作 的安全, 而最终的目的则是保证土地测量工程的质量。在与 人文和生态环境的双重结合过程中, 也能够对相关的生态资 源和生态环境进行合理, 高效率的利用, 与我国现如今生态 文明发展的要求与低碳环保的理念也是不谋而合的, 不仅有 利于提升建筑工程的质量, 也有利于相对应的建筑单位的可 持续性发展。

2.2 数据化处理原图的应用 
数字化测绘技术在工程测量中的主要应用还在于数据 化处理原图的应用, 在整个建筑工程的测量环节中, 数字化 技术对处原图的处理主要表现在扫描失量化与手扶跟踪数 字化这两大方面, 而这两方面对数据的精准度以及操作方式 的高效率等也具有非常严格的要求, 数据化处理技术在保证 准确度这一问题上具有非常明显的优势。但是数据化技术对 原图的处理也具有一定的劣势。由于其在成图的过程中只能 对地表和地表的物体进行一定的描述, 所以其实时性是有待 提高的, 这就要求相关的施工单位要对整个工程现场的实际 信息进行大范围的搜集和整理, 同时与扫描图进行有效的结 合, 才可以对扫描图过程中所形成的误差进行较好的纠正, 从而有效的应对后期整个工程建设中所出现的问题, 这也是 数字化测绘技术在现如今的工程测量中应用的主要目的之 一。

\section{3 在测量定位中的应用}

数字化测绘技术在工程测量的应用之中还有效的表现 在测量定位中的应用。数字化测绘技术不仅能够对数据进行 精准的分析, 从而有效的对后期的实践进行高度的指导, 还 能够在复杂的环境中对测量的地点进行良好的定位, 同时构 建详细的结构信息, 对后期的工程施工等也具有非常明显的 指导作用。而在这一过程中扮演最重要角色的系统是 GPS 系 统。现如今, 科学技术水平得到了大幅度的提高, 同时人工测 量技术也逐渐被取代, GPS 系统在定位中的应用也越来越广 泛。其优势在于 GPS 的应用是不受自然环境等因素的影响的, 这就避免了人为测量中对天气等自然因素的依赖。同时, GPS 系统与人力测量相比, 也具有非常高的精准度, 同时也能够 对卫星所传递的信号进行高效率的接收, 同时对建筑的定位 进行有效的把控, 也有利于建筑施工工程后期的顺利开展。

\section{4 地面数字化测图技术的应用}

在对整个工程进行测量的过程中, 地面数字化的技术往 往会比较倾向于比例尺较大的一些工程项目图的绘制, 那么 在这种情况下, 地面数字化测图技术便得到了大范围的应用, 而这一应用过程中对数据具有较强的依赖性, 而地面数字化 测图技术便能够对这些数据进行大范围的采集, 从而实现高 效率的储存, 然后凭借这些数据对后期的图画绘制进行相对 应的指导。当然, 在这项技术的应用过程中, 图像的高清度也 是十分明显的优势之一, 这与传统的绘图技术相比有着非常
明显的进步。同时也可以有效地提升测量的准确率和效率, 往往只测量一次, 便可以得到非常精准的数据, 从而对后期 的工程施工进行高效率的指导作用。同时, 计算机智能化系 统对数据也可以进行较好的处理, 这样在工程的施工环节中 便可以对工程的工作难度进行大幅度的降低, 而对工程的作 业效率, 实现大幅度的提高。由此可见, 地面数字化测图技术 在工程测量中的应用也是具有十分重要的现实意义的。除此 之外, 在整个工程测量的数据采集这一环节中, 数字化技术 能够对附近的详细数据进行高效率的收集, 同时还能够进行 自动的保存, 这样可以有效的避免人为因素的影响, 从而造 成数据存储不及时等, 影响后期工程的开展。其次, 也可以有 效地降低相对应的测量人员的工作难度和劳动强度, 甚至可 以有效地降低人力资本的投入, 不仅可以有效的减少工程施 工企业内部的人力资源, 还能够对所采集的数据进行精准度 的提高, 可谓是一举两得。企业承包工程施工建设的主要目 的在于盈利, 从而提高整个企业自身的竞争力, 保证企业的 可持续性的发展。减少人力资本的投入, 也可以有效地提高 企业内部的竞争力, 对企业的发展也具有十分重要的辅助作 用。

\section{3 结束语}

近年来, 我国工程行业所取得的发展和进步以及创新性 的工作我们有目共睹, 而科技在工程行业中也得到了更大范 围的融合和创新, 从工程测量这一方面来看, 数字化测绘技 术的应用能够对工程测量这一环节进行高效率的提升, 从而 有效的保证工程施工的质量。而综合的对各类数字化测绘技 术的应用便显得越来越重要, 不仅能够提高工程测量的水平, 还可以为员工的施工安全保驾护航, 更可以直接减少人力资 本的应用, 同时扩大企业内部的经济效益, 对企业的发展具 有非常重要的作用。所以, 保证数字化测绘技术在工程测量 中得到大范围的应用, 也是十分重要的。

\section{[参考文献]}

[1]王殿斌.数字化测绘技术在水利工程测量中的应用 研究[J].居舍,2018(33):65.

[2]胡博.数字化测绘技术在工程测量中的应用研究 [J]. 科技创新与应用,2018(33): 180-181。

[3]王文斌.测绘新技术在测绘工程测量中的应用分析 [J].居舍,2018(32):66. 